We assessed the impact of severity of OSAS on the atrial size and CRP level in patients who were found to have atrial premature complexes.

Methods 277 patients diagnosed with obstructive sleep apnoea after overnight polysomnography underwent a 24-h Holter electrocardiography and ambulatory blood pressure monitoring. 137 patients (49\%) were found to have premature atrial complexes (PAC). These patients were classified into 3 groups according to the apnoea-hypopnoea index (AHI) as mild $(5 \geq \mathrm{AHI}<15)$, moderate $(15 \geq \mathrm{AHI}<30)$ and severe $(\mathrm{AHI} \geq 30)$. Their $\mathrm{C}$-reactive protein level was assessed by a high-sensitive radio-immunoassay. Of the 137 patients with PAC, 77 underwent transthoracic Doppler echocardiography (TTE) and their left atrial diameter (LAD), interventricular septal thickness (IVSD), left ventricle posterior wall thickness (LVPW), left ventricle mass weight (LVMW), peak diastolic early (E) and late (A) transmitral flow were recorded and their respective $\mathrm{E} / \mathrm{A}$ ratio and left ventricular ejection fraction (LVEF) calculated CRP and echocardiographic parameters were compared between the different severity of OSAS patients having premature atrial complexes.

Results There is a high prevalence of premature atrial contraction in OSAS patients'-reactive protein and left atrial diameter was significantly increased with severity of OSAS irrespective of systolic and disastolic function. CRP was higher in severe OSAS (5.01 \pm 4.68$) \mathrm{mg} /$ $\mathrm{L}$ as compared to moderate $(3.03 \pm 1.94) \mathrm{mg} / \mathrm{L}$ and mild OSAS $(2.98 \pm 1.82) \mathrm{mg} / \mathrm{L}$, [mild $\mathrm{v} / \mathrm{s}$ severe OSAS $(\mathrm{p}=0.040) ;$ moderate $\mathrm{v} / \mathrm{s}$ severe $(p=0.033)$ ]; left atrial diameter was more increased in severe OSAS $(40.1 \pm 7.9) \mathrm{mm}$ as compared to moderate $(37.9 \pm 5.5) \mathrm{mm}$ and mild OSAS $(33.7 \pm 3.8) \mathrm{mm}$, [mild $\mathrm{v} / \mathrm{s}$ moderate $(\mathrm{p}=0.025)$, mild $\mathrm{v} / \mathrm{s}$ severe OSAS $(p=0.002)]$.

Conclusion The prevalence of premature atrial contraction (PAC) in OSAS patients is in line with the prevalence of AF in OSAS patients. Severity of OSAS was associated with increase in left atrial size and CRP level in patients with premature atrial complexes. Inflammation associated with OSAS might contribute to atrial structural and electrical remodelling in OSAS patients with premature atrial contraction.

\section{e0561 CLINICAL FACTORS OF INFLUENCING HEART RATE VARIABILITY}

doi:10.1136/hrt.2010.208967.561

${ }^{1}$ Li Ruoru, ${ }^{2}$ Li Fangjie, ${ }^{2}$ Guo Xiaoyu, ${ }^{2}$ Zhai Junpeng. ${ }^{1}$ Shenzhen Traditional Chinese Medicine Hospital; ${ }^{2}$ Wang Jing Hospital, China Academy of Chinese Medical Sciences

Objective To compare the influence of different clinical factors on the HRV time domain, frequency-domain indicators and the strength and type of influence.

Methods A retrospective study on the long-time HRV time domain, frequency domain values of 192 patients with diabetes, coronary heart disease (CHD), hypertension, cancer and cervical spondylosis (CS), to observe the strength and type of each factor when there are multiple factors simultaneously using multiple linear regression statistical method.

Results In different age patients with complications of diabetes, $\mathrm{CHD}$, hypertension, cancer and CS, the significant influencing factors on HRV were age, diabetes and CS. Made SDNN values as the dependent variable, enrolled the final regression equation was in turn age $(\beta=-0.282, p<0.001)$, diabetes $(\beta=-0.237, p<0.001)$ and CS $(\beta=0.151, p=0.024)$; Made SDANN values as the dependent variable, enrolled the final regression equation was also in turn age $(\beta=-0.271, \quad p<0.001)$, diabetes $(\beta=-0.236, \quad p=0.001)$ and CS ( $\beta=0.142, p=0.036$ ); Made LF values as the dependent variable, enrolled the final regression equation was also in turn age $(\beta=-0.364$, $p<0.001)$, diabetes $(\beta=-0.216, p=0.001)$ and $\operatorname{CS}(\beta=0.138, p=0.034)$;
Made TP values as the dependent variable, enrolled the final regression equation was only age $(\beta=-0.285, p<0.001)$ and diabetes $(\beta=-0.174, p=0.011)$; Made HF values as the dependent variable, enrolled the final regression equation was also age $(\beta=-0.277$, $p<0.001)$ and diabetes $(\beta=-0.162, p=0.020)$; which meaning age and diabetes were a negative correlation with SDNN, SDANN, TP and LF, while CS was positively correlated with SDNN, SDANN and LF. Only diabetes had significant influence on RMSSD, it was negatively correlated with RMSSD ( $\beta=-0.148, p=0.040)$.

Conclusion Age increase and having diabetes will significantly reduce the overall tension of autonomic nervous and sympathetic tone, and diabetes can significantly lower vagal tone factor.

\section{e0562 IMPACT OF CIRCUMFERENTIAL PULMONARY VEIN ISOLATION ON ELECTROPHYSIOLOGIC PROPERTIES OF PULMONARY VEINS IN PATIENTS WITH PAROXYSMAL ATRIAL FIBRILLATION}

doi:10.1136/hrt.2010.208967.562

Zhitao Zheng, Yunlong Xia, Lianjun Gao, Shulong Zhang, Donghui Yang, Xiaomeng Yin, Peixin Cong, Dong Chang. First Affiliated Hospital of Dalian Medical University

Objective The special electrophysiological characteristics of pulmonary veins (PVs) are highly correlated with the incidence of Atrial fibrillation (AF), and circumferential PV isolation (CPVI) has been demonstrated to be effective in many patients with paroxysmal AF (PAF). However, the impact of CPVI on electrophysiologic properties of PVs is still rarely known.

Methods Eighteen patients (58.4 \pm 9.3 years old, 9 males) with frequent symptomatic and drug-resistant PAF were enrolled in this study. Under CARTO and Ensite Navx's guidance (13 Carto, 5 Ensite Navx), CPVI was performed in these patients, and totally $72 \mathrm{PV}$ s were isolated, which was confirmed by the disappearing of PV potentials recorded by using the 10-pole circular catheter during sinus rhythm, in the first procedure. Effective refractory period (ERP), Relative Refractory Period (RRP) and Functional Refractory Period (FRP) were measured by use of programmed stimulation with a single extrastimulus in the PVs and left atrial (LA) before and after ablation in each patient. Due to can't record PV potential or cant't pace the PVs after CPVI, the changes of electrophysiologic properties of $33 \mathrm{PVs}$ were used for final analysis.

Results No change of pacing threshold of PVs and LA was found before and after CPVI $(3.2 \pm 0.6 \mathrm{~V}$ vs $3.3 \pm 0.5 \mathrm{~V}$ and $3.3 \pm 0.7 \mathrm{~V}$ vs $3.2 \pm 0.7 \mathrm{~V}$, respectively, $\mathrm{p}>0.05)$. Before CPVI, the ERP and RRP of PVs $(187 \pm 60 \mathrm{msec}$ and $223 \pm 45 \mathrm{msec})$ were significantly shorter than that of the LA $(229 \pm 31 \mathrm{msec}, \quad \mathrm{p}<0.001$ and $261 \pm 34 \mathrm{msec}, \mathrm{p}<0.05)$. After CPVI, the ERP of PVs was significantly increased to $245 \pm 38 \mathrm{msec}$ as compared to that before the ablation $(187 \pm 76 \mathrm{msec}, \mathrm{p}<0.001)$, which became much close to that of LA $(234 \pm 43 \mathrm{~ms}, p>0.05)$. No influence of CPVI on LA ERP was found $(p>0.05)$. The RRP and FRP of PVs were also significantly increased as compared to that before the ablation $(230 \pm 47 \mathrm{msec}$ vs $259 \pm 44 \mathrm{msec}$ and $230 \pm 74 \mathrm{msec}$ vs $298 \pm 47 \mathrm{msec}$, respectively, $\mathrm{p}<0.05$ ), but there were no changes of LA RRP and FRP (256 $\pm 49 \mathrm{msec}$ vs $240 \pm 34 \mathrm{msec}$ and $254 \pm 90 \mathrm{msec}$ vs $276 \pm 47 \mathrm{msec}$, respectively, $\mathrm{p}>0.05$ ).

Conclusions In patients with PAF, an ERP gradience from PVs to LA, and then to RA was observed, with the shortest in PVs and the longest in RA. The CPVI may have different impact on the electrophysiologic properties of PVs and LA. CPVI significantly decrease the arrhythmogenic of PVs and refractory heterogeneity between PVs and LA. These findings may help to explain the maintenance of sinus rhythm in a subgroup of patients with PAF by CPVI in spite of the PVs conduction already recovered. 